\title{
Filtros de Resposta Máxima e Padrões Binários Locais Aplicados na Detecção de Anormalidades em Mamografias
}

\author{
Danilo O. Fistarol ${ }^{1}$ \\ Wesley Nunes Gonçalves ${ }^{1}$ \\ ${ }^{1}$ Universidade Federal do Mato Grosso do Sul - Campus Ponta Porã \\ Rua Itibiré Vieira, s/n - Residencial Julia Oliveira Cardinal BR 463 - Km 4,5 79.907-414 \\ Ponta Porã - MS - Brasil \\ \{danilofistarol, wnunesgoncalves\}@gmail.com
}

\begin{abstract}
Breast cancer is a malignant tumor that develops as a result of genetic changes in a group of breast cells. In this paper we use texture analysis, one of the sub-areas of computer vision, to assist in the detection of probable breast lumps. The assumption is that the texture of lumpy regions differs from the texture of normal regions. In texture analysis, a technique that stands out is the local binary patterns (LBP). Although LBP provides good results in texture images, its performance is drastically low in mammography images. To improve the performance, this paper proposes the use of the maximum response filters and LBP for detecting abnormalities in mammograms. The proposed method achieved an accuracy of $88 \%$ compared to only $68 \%$ of LBP.
\end{abstract}

Resumo. O câncer de mama é um tumor maligno que se desenvolve como consequência de alterações genéticas em um conjunto de células da mama. Neste trabalho utilizamos a análise de textura, uma das subáreas da visão computacional, para auxiliar na detecção de prováveis nódulos mamários. A suposição é que a textura de regiões com nódulos seja diferente da textura de regiões normais. Em análise de textura, uma técnica que se destaca são os padrões binários locais (LBP). Embora o LBP obtenha bons resultados em imagens de textura, seu desempenho foi drasticamente baixo em imagens de mamografia. Para contornar esse problema, esse artigo propõe o uso dos filtros de resposta máxima e o LBP para a classificação de nódulos em mamografias. O método proposto obteve uma acurácia de $88 \%$ comparado com apenas $68 \%$ do LBP.

\section{Introdução e Justificativa}

O câncer de mama é o segundo tipo de câncer mais frequente no mundo. Entre as mulheres, este é o câncer mais comum, correspondendo a $25 \%$ dos casos em 2012, com aproximadamente 1,7 milhões de vítimas, sendo a quinta causa de morte por câncer em geral [INCA 2014]. Apesar do alto índice, se diagnosticado e tratado oportunamente, o prognóstico de cura é relativamente bom. No Brasil, as taxas de mortalidade por câncer de mama continuam elevadas, provavelmente porque a doença ainda é diagnosticada em estágios avançados. Embora o câncer seja considerado uma doença do mundo desenvolvido devido ao estilo de vida, a maioria (69\%) das mortes por esta causa são registrados em países em desenvolvimento [OMS 2014]. 
A mamografia por raio $\mathrm{X}$ ainda é o exame mais adotado para detecção precoce de sinais de câncer de mama e tem um papel importante nas decisões médicas a serem adotadas. A mamografia por raio X pode revelar evidências de anormalidades como nódulos e calcificações, bem como sinais sutis de assimetria bilateral e distorção arquitetural [Rangayyan et al. 2007]. No entanto, esta modalidade de exame apresenta limitações para detectar tumores malignos ocultos em mamas densas ou em mamas com implante, devido a sobreposição de tecidos causada pela projeção bidimensional da mama.

Como alternativa para uma segunda opinião ou como uma estratégia de pré-leitura, sistemas de apoio à detecção e diagnóstico (CAD) de câncer de mama têm sido propostos [Thurfjell et al. 1994, Verma and Zakos 2001]. Estes sistemas apresentam melhor sensibilidade no diagnóstico, sem um aumento significativo de taxa de erro. Em geral, um sistema CADe envolve etapas de pré-processamento, detecção de regiões candidatas (suspeitas) a lesão, e redução de falsos positivos. O pré-processamento da imagem é uma etapa importante em sistemas CADe, cujo objetivo é a eliminação de ruídos e artefatos que podem dificultar a detecção de uma região suspeita e/ou causar alta taxa de falsos positivos. Entretanto, a etapa de detecção é o estágio mais crítico e importante no sistema.

Neste trabalho pretende-se utilizar uma das subáreas da visão computacional para auxiliar na detecção de prováveis nódulos mamários, a análise de textura. O conceito de textura está relacionado com o arranjo espacial dos níveis de cinza entre os pixels de uma dada região na imagem. Portanto, a análise de textura tem como objetivo extrair informações da imagem que caracterize a distribuição dos níveis de cinza. A proposta é que a textura de regiões contendo nódulos seja diferente da textura de regiões normais, e estas diferenças sejam capturadas por métodos de textura.

A técnica de textura Padrões Binários Locais (Local Binary Patterns - LBP) [Ojala et al. 1996] tem sido utilizada com grande sucesso na extração de características de textura, por possuir baixo custo computacional, ser invariante a iluminação, rotação e à escala quando normalizado. No entanto, ao ser aplicada em imagens de mamografia, a técnica não obtém um bom desempenho. Para aprimorar a caracterização de um nódulo, este artigo propõe o uso dos filtros de Resposta Máxima (Maximum Response - MR8) [Varma and Zisserman 2003] para evidenciar as características relevantes das amostras seguida pela extração de características com o LBP. Nos experimentos, as imagens da base foram classificadas em duas classes: regiões normais e anormais. O método proposto obteve uma porcentagem de acerto de $88 \%$ enquanto que o LBP obteve $68 \%$, uma melhora de $20 \%$ na detecção de regiões anormais.

As demais seções do artigo estão organizadas da seguinte maneira: Na Seção

2 é feita uma revisão bibliográfica das técnicas de texturas utilizadas na literatura; $\mathrm{Na}$ Seção 3 é descrito como foi o processo de aquisição das imagens e o método proposto; Os experimentos e resultados obtidos são apresentados na Seção 4 e, por fim, a Seção 5 apresenta as conclusões e os trabalhos futuros.

\section{Revisão de Literatura}

Desde que Wilhelm Röentgen, em 1895, descobriu a existência de uma radiação capaz de atravessar materiais, o raio $X$ tornou possível a visualização de estruturas internas do corpo e o diagnóstico de anomalias. A partir de então, o avanço na aquisição, processamento e armazenamento de imagens médicas vem permitindo o aperfeiçoamento 
de diagnósticos e tratamentos de doenças de diversas naturezas, entre elas o câncer de mama. A mamografia por raios-X pode evidenciar anormalidades como nódulos e calcificações, bem como sinais sutis de assimetria bilateral e distorção arquitetural [Rangayyan et al. 2007].

$\mathrm{Na}$ literatura, alguns pesquisadores propuseram algoritmos para detectar massas em mamografias. [Vyborny and Giger 1994] discutiram algoritmos para detectar anormalidades tais como: massa e micro calcificação de mamografias utilizando sistemas CAD. Eles concluíram que, para uma comparação justa, os algoritmos devem ser avaliados em um banco de dados público. No entanto, na ausência de qualquer base de dados, os algoritmos existentes deveriam comparar seus resultados diretamente com a avaliação do radiologista. Em 1994, Mammographic Image Analysis Society [Suckling et al. 1994] lançou um banco de dados público composto por 322 filmes de mamografias digitalizadas. Posteriormente, outro banco de dados conhecido como o Digital Database for Screening Mammography (DDSM) [Heath et al. 1998] foi publicado para fins de pesquisa. Estas e outras bases de dados têm ajudado os pesquisadores a identificar e resolver alguns problemas chave associados ao desenvolvimento de sistemas CAD para mamografias.

Em relação à caracterização de imagens, [Mudigonda et al. 2000] propuseram dois métodos de extração de caraterísticas baseados em matriz de coocorrência e na transformação de densidade ótica para descrever caraterísticas locais da textura e a distribuição fotométrica discreta de cada região de interesse (ROI). Para a classificação entre massa Benigna e Maligna, obteve-se $82.1 \%$ de acurácia. Para a detecção de massa, a segmentação de mamografias gera amostras que podem representar não apenas as áreas de massa, mas os tecidos normais, isto resulta no problema de falsos positivos, ou seja, classificação de tecidos normais como massa. [Hussain 2013] trabalha com esse problema utilizando um descritor de textura local com base em um banco de filtros de Gabor que é utilizada para representar as amostras em massa.

A técnica de extração de características a ser utilizada neste trabalho é o Padrões Binários Locais (LBP) [Ojala et al. 1996], que vem sendo utilizada como um descritor robusto para obtenção de informações relacionados a textura de uma imagem. As principais vantagens são a não influência das variações de iluminação, a baixa complexidade computacional, a capacidade de codificar detalhes e permitir realizar análise local das informações. No trabalho de [Kitanovski et al. 2011] é feita uma comparação entre métodos de extração de características e classificadores. Foram utilizados cinco métodos de extração: LBP, Diferença de Níveis de Cinza (GLDM), Comprimento de Primitivas de Níveis de Cinza (GLRLM), Haralick e Gabor. Os algoritmos de classificação utilizados foram o K-Vizinhos mais próximos - Knn, a Máquina de Vetores de Suporte - SVM e o algoritmo C4.5, que constrói árvores de decisão. De todos os métodos e classificadores, o que obteve melhor resultado foi o método GLDM no classificador Knn, tendo uma acurácia de $98.77 \%$, enquanto a técnica LBP, obteve $95.38 \%$ com o classificador SVM. [Liu et al. 2011] aplica a técnica LBP, com uma modificação de pesos nos vizinhos, para classificar nódulos benignos e malignos em mamografias, na etapa de classificação foi utilizado o SVM, obtendo $66.15 \%$ de acerto. [Nanni et al. 2012] utilizando a base DDSM compara diferentes tipos descritores de textura para classificar os nódulos em mamografias como benignos ou malignos, tendo $88.6 \%$ de acurácia utilizando o método LBP e o classificador SVM. [Duarte et al. ] utiliza a técnica LBP e uma variação da mesma para 
classificar lesões em mamografias, a fim de evidenciar informações que caracterizem melhor a imagem é utilizada a transformada wavelet, obtendo uma acurácia de 79,17\% no método LBP para a classificação de nódulos normais e anormais.

Para evidenciar características de imagens, [Varma and Zisserman 2003] propuseram 38 filtros de Resposta Máxima (MR8). A imagem é convoluída com os filtros e então os valores máximos são mantidos para que ao final dessa etapa sejam geradas 8 imagens filtradas. Neste trabalho, os filtros MR8 são aplicados em imagens de mamografia com o intuito de evidenciar as características importantes para que a caracterização pelo LBP seja mais eficaz.

\section{Metodologia}

Nesta seção, é descrita a metodologia que inclui a aquisição e pré-processamentos das amostras das classes normais e anormais e o método proposto para caracterização das mesmas.

\subsection{Aquisição de Imagens}

As imagens utilizadas neste trabalho fazem parte da base de dados MIAS (mini Mammographic Image Analysis Society), que contém imagens de mamografias de raio X com tamanho original de $1024 \times 1024$ pixels. A partir dessa base, foram selecionadas aleatoriamente 100 imagens, sendo 50 normais e 50 com nódulos.

Para imagens que possuem algum tipo de nódulo foi feita a seleção da amostra com base nas informações de localização que um especialista forneceu na base. O tamanho das amostras está relacionado com o diâmetro do nódulo, sendo que o tamanho mínimo para as imagens foi $50 \times 50$ pixels. Na Figura 1(a) são exibidas amostras da classe anormal que nódulos. Para as mamografias pertencentes à classe normal foi feita a seleção aleatória das amostras, com um tamanho de $50 \times 50$ pixels, como pode ser visto na Figura 1(b). Por conter amostras de tamanho diversos, esta base torna-se um desafio com relação a invariância a escala e rotação.
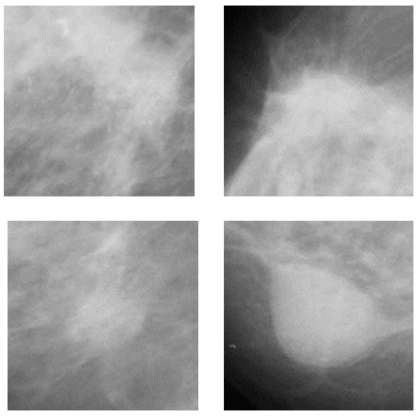

(a) Amostras da classe Anormal
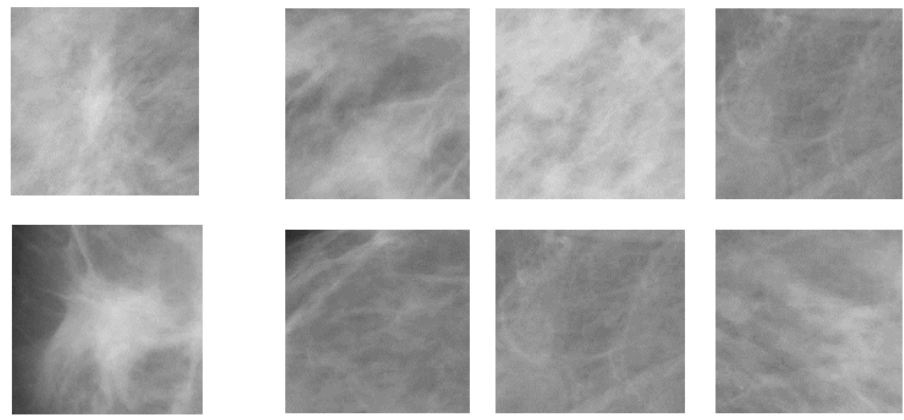

(b) Amostras da classe Normal

Figura 1: A base utilizada neste trabalho possui 100 amostras, divididas em 50 Normais e 50 Anormais, com o tamanho mínimo de $50 \times 50$ pixels.

\subsection{Método Proposto}

O método proposto pode ser descrito em duas etapas: convolução da amostra com os filtros de resposta máxima (MR8) e extração de características com os padrões binários 
locais. Na primeira etapa, a amostra é convoluída com os filtros MR8 com o intuito de evidenciar as características. O banco de filtros MR8 é composto por 38 filtros de borda, barra e Gaussiana conforme ilustrado na Figura 2(a). Os filtros de borda e barra ocorrem em várias orientações, mas suas respostas são unidas a fim de armazenar somente a resposta máxima em todas as orientações. Devido a esse procedimento, a resposta do filtro é invariante a rotação, o que é uma propriedade desejável para os métodos de textura.

A Figura 2(a) apresenta os 38 filtros onde os dois últimos são, respectivamente, o filtro Gaussiano e o Laplaciano da Gaussiana com $\sigma=10$ pixels. Ambos os filtros possuem simetria de rotação. Os demais filtros correspondem aos filtros de borda orientados em 3 escalas $\left(\left(\sigma_{x}, \sigma_{y}\right)=\{(1,3),(2,6),(4,12)\}\right)$ e os filtros de barra orientados nas mesmas escalas. Para cada escala, os filtros ocorrem em 6 orientações e, medindo apenas a resposta máxima em todas as orientações, as respostas são reduzidas de 38 (6 orientações, 3 escalas para dois filtros orientados, e 2 Gaussianos) para 8 (3 escalas para 2 filtros, e 2 Gaussianos). Portanto, ao final da primeira etapa, para uma amostra são geradas oito novas, como pode ser visto na representação feita na Figura 2(b), onde a figura central é a amostra original e as demais são as 8 respostas.

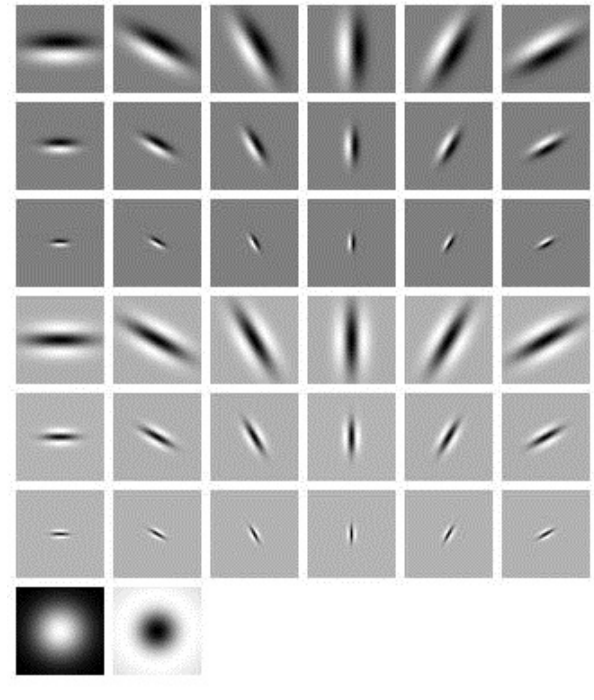

(a) Banco de filtros de resposta máxima.

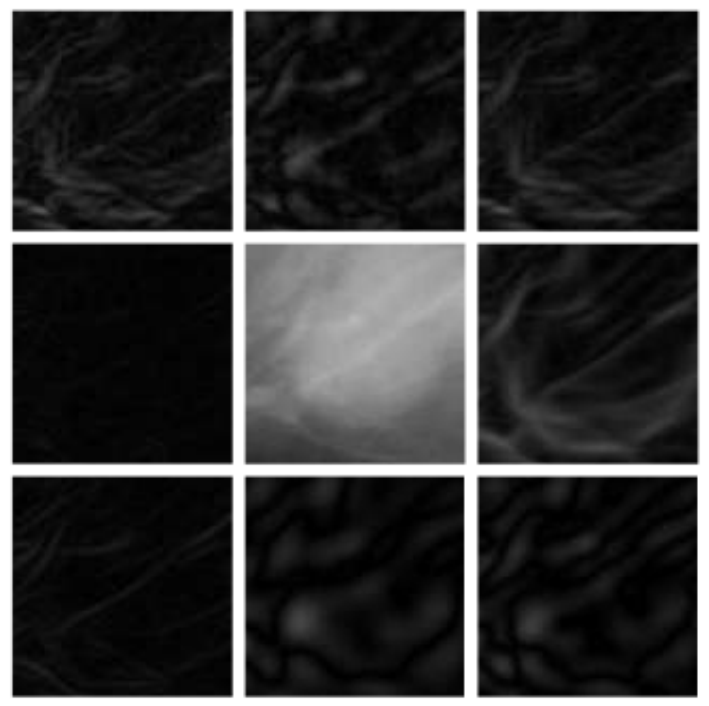

(b) Amostras após aplicação dos filtros MR8.

\section{Figura 2: Banco de 38 filtros de resposta máxima (MR8) - Os 36 primeiros fil- tros são orientados, em três escalas e seis orientações diferentes, os dois últimos são os filtros isotrópicos.}

Na segunda etapa, os padrões binários locais são aplicados em cada uma das 8 respostas para extração de características de textura. No LBP, um código binário é calculado para cada pixel por meio da comparação da sua intensidade com a intensidade de $\mathrm{P}$ vizinhos localizados a um raio $\mathrm{R}$ de distância, formando um círculo ao redor do pixel. Se a intensidade do pixel central for maior que a intensidade de um dado vizinho, então o valor 0 é atribuído, caso contrário, o valor 1, como pode ser observado na Figura 3. As atribuições para os $\mathrm{P}$ vizinhos fornecem um número binário de $\mathrm{P}$ dígitos, que é convertido para decimal. Por exemplo, o número binário obtido no exemplo da Figura 3 é 11110001 , que é convertido para o decimal 241. Portanto, cada pixel da imagem fornece um número decimal que são, por fim, agrupados em um histograma que descreve a textura presente 
na imagem.

\begin{tabular}{|c|c|c|c|c|}
\hline 129 & 255 & 198 & 122 & 90 \\
\hline 243 & 212 & 192 & 176 & 100 \\
\hline 120 & 122 & 156 & 222 & 213 \\
\hline 54 & 67 & 111 & 216 & 255 \\
\hline 100 & 212 & 192 & 255 & 122
\end{tabular}$\quad$ LBP \begin{tabular}{|c|c|c|c|}
\hline 1 & 1 & 1 \\
\hline 0 & & 1 \\
\hline 0 & 0 & 1 \\
\hline
\end{tabular}

Figura 3: Processo de comparação do pixel central com seus vizinhos.

O LBP tradicional, conforme descrito anteriormente, extrai um vetor de característica com dimensão $\Re^{2^{P}}$. Para evitar o mau da dimensionalidade que ocorre para vetores com muitas características, este trabalho utiliza uma variante do LBP chamada LBP uniforme. Em vez de utilizar a conversão de binário para decimal, após a etapa de comparação com os vizinhos, o LBP uniforme calcula o número de mudanças que ocorrem dos valores 1 para 0 ou vice versa. Se houver duas ou menos variações, o pixel é dito uniforme. Para pixels uniformes, seu código é dado pela soma de todos os 1, enquanto pixels não uniformes possuem código fixo igual $P+1$. No exemplo da Figura 3, o pixel é dito uniforme e seu código é 5. Por fim, calcula-se o histograma com os códigos de todos os pixels. Dessa forma, o vetor de características final possui dimensão $\Re^{P+2}$.

O método proposto pode ser sumarizado na Figura 4. Dada uma amostra, esta é convoluída com os filtros MR8 fornecendo 8 amostras com as características evidenciadas. Para cada amostra convoluída $i$, o LBP uniforme é aplicado com três valores de vizinhos e raios $(P, R):(8,1),(16,2),(24,3)$, obtendo-se três vetores de características $\varphi_{8,1}^{i}, \varphi_{16,2}^{i}, \varphi_{24,3}^{i}$. A textura de cada amostra convoluída é representada por um vetor de características composto pela concatenação: $\varphi^{i}=\left[\varphi_{8,1}^{i}, \varphi_{16,2}^{i}, \varphi_{24,3}^{i}\right]$. Por fim, os vetores de todas as amostras convoluídas são concatenados para formar o vetor de características final, $\varphi=\left[\varphi^{i}\right], 1 \leq i \leq 8$.

\section{Experimentos e Resultados}

Para avaliar a eficácia das técnicas de extração de características é necessário o uso de um classificador. Nesta trabalhos, três classificadores foram utilizados: K-Vizinhos mais próximos (k-nearest neighbor - Knn) [Duda et al. 2012], Naive Bayes [Zhang 2004] e Máquina de Vetores de Suporte (Support Vector Machines - SVMs). Para os três algoritmos de classificação foi utilizado a amostragem de validação cruzada com 10 pastas. Nesta amostragem, as imagens são divididas aleatoriamente em 10 partições mutuamente exclusivas. Assim, nove partições são utilizadas para o treinamento do classificador e uma partição é utilizada para o teste. O processo se repete 10 vezes de modo que cada partição seja utilizada uma vez para testar o classificador. Ao final desta etapa, é possível obter a taxa de acurácia com base na média dos acertos calculados em cada uma das partições.

A Tabela 1 apresenta a comparação entre o método proposto e os Padrões Binários Locais com os três classificadores na base com classes Normal e Anormal. É possível observar que o método proposto e o LBP obtiveram o melhor resultado com o classificador SVM (em negrito). Para este classificador, o método proposto e o LBP obtiveram $88 \%( \pm 10.36)$ e $67 \%( \pm 14.57)$, respectivamente. Esses resultados mostram a eficácia do 


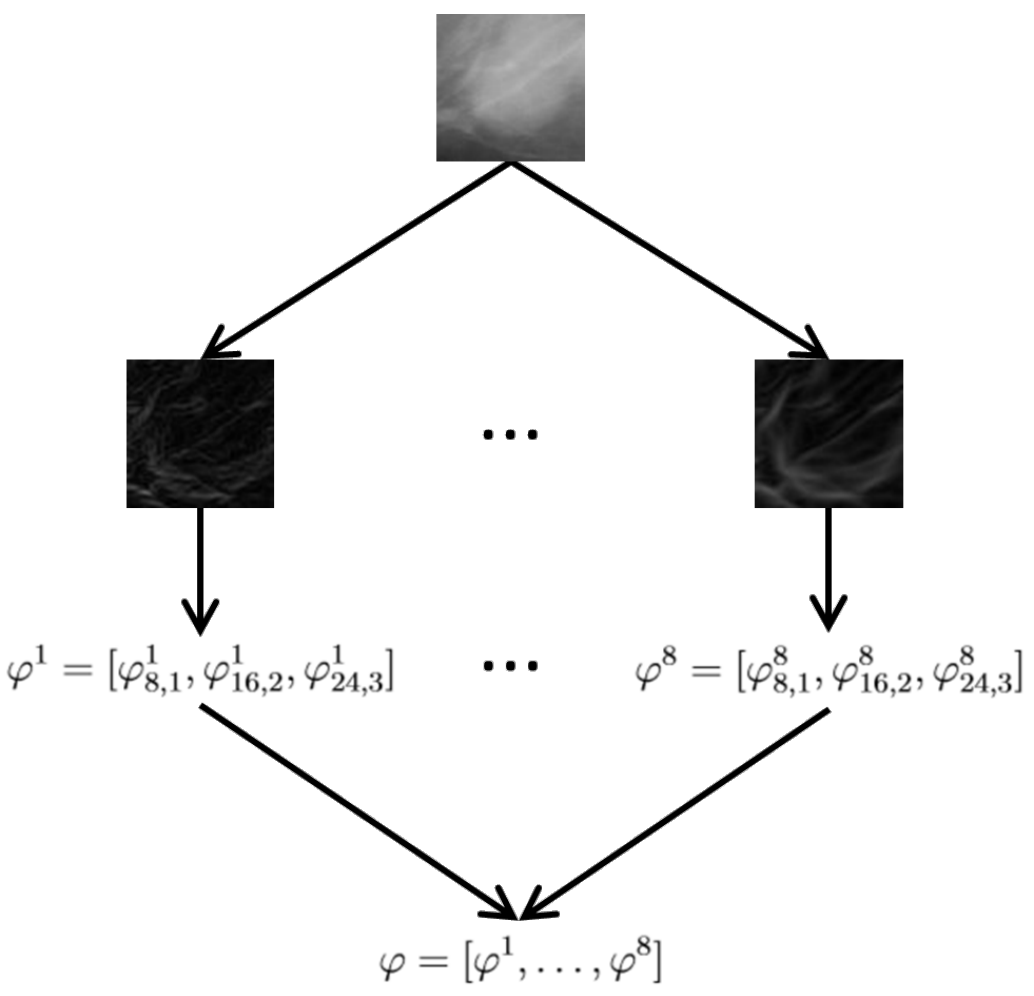

Figura 4: Diagrama do método proposto. 0 método proposto recebe como entrada a amostra, que é convoluída com os filtros MR8. Então as características são extraídas pelo LBP que gera o um vetor de característica para cada amostra. Por fim, os 8 vetores são concatenados em um único que representa a amostra na etapa de classificação.

método proposto, que obteve um aumento de $21 \%$ na classificação de imagens de mamografia. Portanto, é possível concluir que os filtros de Resposta Máxima auxiliam e evidenciam as características.

\begin{tabular}{|c|c|c|}
\cline { 2 - 3 } \multicolumn{1}{c|}{} & LBP & Método Proposto \\
\hline Classificador & \% (Desvio Padrão) & \% (Desvio Padrão) \\
\hline KNN & $63.00( \pm 8.93)$ & $51.00( \pm 4.19)$ \\
\hline Naive Bayes & $66.00( \pm 14.45)$ & $85.00( \pm 12.57)$ \\
\hline SVM & $\mathbf{6 7 . 0 0}( \pm \mathbf{1 4 . 5 7})$ & $\mathbf{8 8 . 0 0}( \pm \mathbf{1 0 . 3 6})$ \\
\hline
\end{tabular}

Tabela 1: Resultados obtidos pelo método proposto e os padrões binários locais usando três classificadores.

Uma etapa muito utilizada em processamento de imagens é a equalização com o intuito de melhorar o contraste das amostras. Para verificar se o LBP e o método proposto se beneficiam com esse pré-processamento, a Tabela 2 apresenta os resultados da classificação das amostras equalizadas com a técnica de equalização global do histograma. Para o LBP, o melhor resultado de $68 \%( \pm 14.16)$ foi obtido usando o Naive Bayes como classificador, o que indica o aumento de $1 \%$ com relação as imagens não equalizadas. $\mathrm{O}$ método proposto obteve seu melhor resultado de $87 \%( \pm 12.07)$ também para o classificador Naive Bayes. Embora muito similar, o resultado foi inferior se comparado com as imagens não normalizadas. Esse resultado se deve ao fato dos filtros de Resposta Máxima 
já melhorarem o contraste além de evidenciarem as principais características das amostras.

\begin{tabular}{|c|c|c|}
\cline { 2 - 3 } \multicolumn{1}{c|}{} & LBP & Método Proposto \\
\hline Classificador & \% (Desvio Padrão) & \% (Desvio Padrão) \\
\hline KNN & $61.00( \pm 10.68)$ & $51.00( \pm 3.49)$ \\
\hline Naive Bayes & $\mathbf{6 8 . 0 0}( \pm \mathbf{1 4 . 1 6})$ & $\mathbf{8 7 . 0 0}( \pm \mathbf{1 2 . 0 7})$ \\
\hline SVM & $64.00( \pm 13.52)$ & $86.00( \pm 10.67)$ \\
\hline
\end{tabular}

Tabela 2: Resultados obtidos pelo método proposto e os padrões binários locais usando três classificadores e amostras equalizadas pela técnica de equalização global do histograma.

Por fim, a Figura 5 apresenta e compara as matrizes de confusão da melhor classificação do LBP (68\% usando amostras equalizadas e classificador Naive Bayes) e do método proposto ( $88 \%$ usando o classificador SVM). Podemos observar que o LBP classifica as amostras anormais com uma boa porcentagem (43 de 50), entretanto, o desempenho cai drasticamente para a classificação de amostras normais (25 de 50). Por outro lado, o método proposto mantém um bom desempenho para ambas as classes normais (43 de 50) e anormais (45 de 50).

\begin{tabular}{|c|c|c|c|c|c|}
\hline & Anormal & Normal & & Anormal & Normal \\
\hline Anormal & 43 & 07 & Anormal & 45 & 05 \\
\hline Normal & 25 & 25 & Normal & 07 & 43 \\
\hline
\end{tabular}

Figura 5: Matriz de confusão referente a classificação das amostras sem a etapa dos filtros MR8 (68\%) e com a etapa dos filtros (88\%).

\section{Conclusão}

Neste trabalho foi proposto um método para detecção de anormalidades em mamografias baseado em filtros de resposta máxima e padrões binários locais. Para classificação entre amostras normais e anormais foram utilizados os classificadores SVM, KNN e Naive Bayes. Na etapa de classificação os melhores resultados foram obtidos pelo método proposto sem a etapa de equalização, tendo $88 \%$ de acurácia com o SVM enquanto que a acurácia para as amostras equalizadas foi de $87 \%$ com Naive Bayes. Por outro lado, utilizando o método LBP, a maior acurácia foi de apenas $68 \%$ utilizando amostras equalizadas. Pode-se enfim considerar que o método proposto aumenta consideravelmente a caracterização de anormalidades em mamografias.

Em trabalhos futuros, pretende-se utilizar outras técnicas de extração de características como, por exemplo, dimensão fractal, matriz de co-ocorrência e filtros de Gabor, além de utilizar uma base com mais amostras e outros filtros de Resposta Máxima.

\section{Agradecimentos}

Ao MEC/SESu, através do Programa de Educação Tutorial (PET/Fronteira), no qual o primeiro autor deste artigo é bolsista. 


\section{Referências}

Duarte, Y. A., do Nascimento, M. Z., Duarte, S. C., and Neves, L. A. Evaluating lbp and clbp in classifying of mammograms lesions.

Duda, R. O., Hart, P. E., and Stork, D. G. (2012). Pattern classification. John Wiley \& Sons.

Heath, M., Bowyer, K., Kopans, D., Kegelmeyer Jr, P., Moore, R., Chang, K., and Munishkumaran, S. (1998). Current status of the digital database for screening mammography. In Digital mammography, pages 457-460. Springer.

Hussain, M. (2013). False positive reduction using gabor feature subset selection. In Information Science and Applications (ICISA), 2013 International Conference on, pages 1-5. IEEE.

INCA (2014). http://www2.inca.gov.br/wps/wcm/connect/acoes_programas/site/home/nobrasil/ programa_controle_cancer_mama/conceito_magnitude. Access date: 27 nov. 2014.

Kitanovski, I., Jankulovski, B., Dimitrovski, I., and Loskovska, S. (2011). Comparison of feature extraction algorithms for mammography images. In Image and Signal Processing (CISP), 2011 4th International Congress on, volume 2, pages 888-892. IEEE.

Liu, J., Liu, X., Chen, J., and Tang, J. (2011). Improved local binary patterns for classification of masses using mammography. In Systems, Man, and Cybernetics (SMC), 2011 IEEE International Conference on, pages 2692-2695. IEEE.

Mudigonda, N. R., Rangayyan, R. M., and Desautels, J. L. (2000). Gradient and texture analysis for the classification of mammographic masses. Medical Imaging, IEEE Transactions on, 19(10):1032-1043.

Nanni, L., Lumini, A., and Brahnam, S. (2012). Survey on lbp based texture descriptors for image classification. Expert Systems with Applications, 39(3):3634-3641.

Ojala, T., Pietikäinen, M., and Harwood, D. (1996). A comparative study of texture measures with classification based on featured distributions. Pattern recognition, 29(1):5159.

OMS (2014). http://www.who.int/topics/cancer/breastcancer/es/index1.html. Access date: 27 nov. 2014.

Rangayyan, R. M., Ayres, F. J., and Leo Desautels, J. (2007). A review of computeraided diagnosis of breast cancer: Toward the detection of subtle signs. Journal of the Franklin Institute, 344(3):312-348.

Suckling, J., Parker, J., Dance, D., Astley, S., Hutt, I., Boggis, C., Ricketts, I., Stamatakis, E., Cerneaz, N., Kok, S.-L., et al. (1994). The mammographic image analysis society digital mammogram database.

Thurfjell, E. L., Lernevall, K. A., and Taube, A. (1994). Benefit of independent double reading in a population-based mammography screening program. Radiology, 191(1):241-244.

Varma, M. and Zisserman, A. (2003). Texture classification: Are filter banks necessary? In Proceedings of the IEEE Conference on Computer Vision and Pattern Recognition, volume 2, pages 691-698. 
Verma, B. and Zakos, J. (2001). A computer-aided diagnosis system for digital mammograms based on fuzzy-neural and feature extraction techniques. Information Technology in Biomedicine, IEEE Transactions on, 5(1):46-54.

Vyborny, C. J. and Giger, M. L. (1994). Computer vision and artificial intelligence in mammography. AJR. American journal of roentgenology, 162(3):699-708.

Zhang, H. (2004). The optimality of naive bayes. $A A, 1(2): 3$. 